\title{
Sound Diffraction Over Noise Barriers with Added Devices Installed on the Top Edge
}

\author{
J. PIECHOWICZ \\ AGH University of Science and Technology, al. Mickiewicza 30, 30-059 Krakow, Poland
}

\begin{abstract}
Airborne sound insulation index of the noise barrier panel and the sound absorption coefficient of the barrier surface are the acoustic parameters that are usually determined in specialized laboratories, however they can be also determined in situ. Acoustic characteristics of a barrier include also the diffraction index difference determined from comparison of barriers with plain top edges and barriers with added devices installed on the top edge. The index is determined from the impulse response values determined for the acoustic wave propagation path from the sound source to a set of properly distributed measurement points. By means of the same method, one can also determine the difference in a barrier's acoustic effectiveness between the plain top barrier structure and its version with added devices mounted on the top. The paper presents measurement results for three types of added devices mounted on the top edge of the barrier. The diffraction index differences have been determined for each added device type and the acoustic effectiveness for each device has been compared with the plain top edge acoustic barrier.
\end{abstract}

DOI: 10.12693/APhysPolA.128.A-36

PACS: 43.20.El, 43.20.Fn, 43.50.Gf, 43.50.Yw, 43.60.Ek, 43.55.Rg

\section{Introduction}

Acoustic characteristics of noise barrier panels, such as the acoustic insulation power or the sound absorption coefficient, are usually determined in reverberation chambers in the diffused acoustic field conditions. The noise barrier as a noise-abatement road device is usually applied in the open space, in quasi-free field conditions. The abundance of noise barriers is not always justified by technical reasons for their application. Each sound barrier is a costly installation which often brings in a disharmony into the local landscape [1] and when installed without a proper technical justification, it simply constitutes an excessive element. In the current technical solutions, such extra elements called added devices are installed on the top edge of barriers. According to designers, they are supposed to improve acoustic properties of the barriers in comparison to barriers with plain top edges. The performance of new elements, based on modified diffraction of the acoustic wave, has been the subject of experimental studies described in the present work. The paper is related to the author's previous work $[2,3]$ in which determination of the diffraction modification evaluation index has been described for an octagonal added device.

Directly behind the barrier, the nature of the sound wave manifests itself by the effects of wave diffraction and interference. Acoustic waves reach the observation point from many various directions, with various amplitudes and various phase relations (see Fig. 1). Because of the acoustic wave diffraction effects occurring on the barrier edges, the sound propagation path depends on the frequency of the propagating sound.

The acoustic wave diffraction phenomena taking place on the top edge of the barrier are described e.g. in [2], where results of effectiveness studies are also shown for octagonal device mounted on the barrier edge.

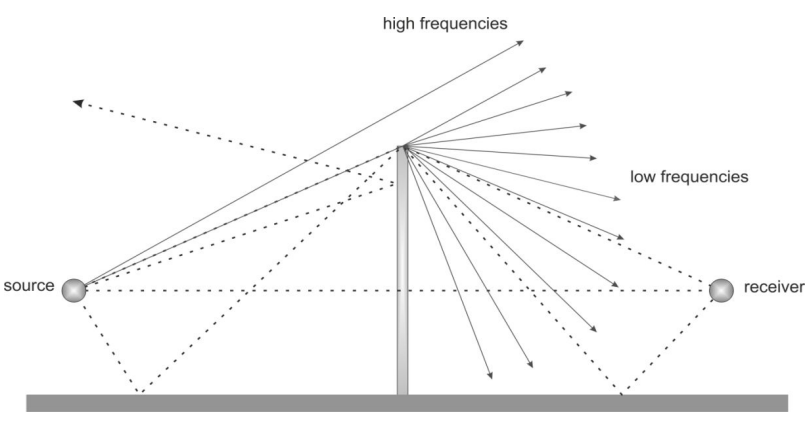

Fig. 1. Sound propagation paths in the presence of noise barrier.

The determined diffraction index difference $(\Delta D I=0)$, has indicated that there was no improvement in the acoustic properties of the barrier when comparing the barrier with and without the added device. Oldham and Egan in their work [4] present results of studies applying modeling of the acoustic field behind a noise barrier using the boundary elements method (BEM) and performing verification of the simulated field parameters as well as assessment of effectiveness for various edge devices of ' $\mathrm{T}$ ', ' $\mathrm{Y}$ ', and $\uparrow$ ('arrow') shapes by means of measurements. In the discussion it has been shown that for the barriers with reflecting surface panels, the barrier effectiveness can be described using the propagation path differences, and in majority of environmental conditions, the best acoustic parameters are achieved with the ' $\mathrm{T}$ ' profile. The distributions of acoustic field parameters in the area near the sound barrier can be studied by sound pressure $[2,5]$ or sound intensity methods [6]. It is of particular importance when verification of numerical modeling is required. The problems regarding improvement of the barrier's acoustic properties resulting from application of added edge devices installed on the top 
edge of the barrier are still among the current problems awaiting a solution. The respective studies are carried out in many world research centers e.g. in Japan [5], Italy [7, 8], United Kingdom [9], and other countries [1013]. In the investigated problems, improvement of acoustic effectiveness of the barrier is not the only important task. The application of added devices on the barrier edges includes also related problems concerning the barrier resistance to weather conditions as well as accumulation of physical and chemical pollutants characteristic for the road traffic.

\section{Diffraction effects on the edge of the noise barrier}

Diffraction is the dominating process affecting the sound wave propagation in the area surrounding the noise barrier. Without including the diffraction effects in the modeling of acoustic fields, one can arrive at numerical results that are inconsistent with values obtained from experimental research. Applying the theoretical basis of GDT (Geometrical Diffraction Theory) [14], one can compare the calculated values of sound level with the values obtained from the experiment for a selected set of observation points located behind the barrier.

In the experimental research, a frequently implemented method is the impulse response method applied to sound wave transmission paths over the top of the sound barrier. The test signal used in this method is usually the MLS (Maximum Length Sequence) in order to have good $\mathrm{S} / \mathrm{N}$ ratios. In the presented experimental studies, the research has been focused on differences between the impulse responses for the wave propagation in the free field and over the top edge of the barrier by applying the dependence of the sound level difference $\Delta L(f)$ for a given frequency $f$ and a given observation point for acoustic wave transmission across the space with and without the sound barrier,

$$
\Delta L(f)=-10 \log \left(\frac{H_{\mathrm{dk}}(f)}{H_{\mathrm{i}}(f)} \frac{d_{\mathrm{dk}}}{d_{\mathrm{i}}}\right)^{2} \mathrm{~dB},
$$

where $H_{\mathrm{dk}}$ is the impulse response for a wave diffracted over the top edge of the barrier, $H_{\mathrm{i}}$ - the impulse response for a wave in a free field, $d_{\mathrm{dk}}$ - distance between the loudspeaker and the observation point for the diffracted wave, and $d_{\mathrm{i}}$ - distance between the loudspeaker and the observation point for the direct wave.

The diffraction index differences $\Delta D I[15]$ have been determined for various shapes of the added devices installed on the top edge of the noise barriers using procedure described in $[2,15]$. In one series of measurements, the reference impulse responses have been determined for free field transmission and two arrangements of the loudspeaker-microphone system. The modification of acoustic properties of the noise barrier comprises mainly the changes in diffraction conditions on the top edge of the barrier. When comparing the added device's effect on the acoustic wave propagation behind the barrier, the diffraction index $D I_{0}$ is determined for the barrier without the added device and then diffraction index $D I_{\mathrm{ad}}$ is determined with the devices mounted on the top edge. The diffraction index difference $\Delta D I$ is the difference between the measurement results obtained for a given barrier construction with the added device $\left(D I_{\mathrm{ad}}\right)$ and without the device mounted on the edge $\left(D I_{0}\right)$ :

$$
\Delta D I=D I_{\mathrm{ad}}-D I_{0} \text {. }
$$

A single-number rating of the diffraction index difference $D I_{\triangle D I}$ characterizes the change in the acoustic wave propagation conditions for the wave transmitted over the top edge of the barrier with the added device and the same barrier with a plain top edge. The index is determined in reference to the frequency spectrum, in octave frequency bands routinely applied to the normalized road traffic noise spectrum [15]:

$$
D I_{\Delta D I}=-10 \log \left(\frac{\sum_{n=1}^{18} 10^{0.1 L n} 10^{-0.1 \Delta D I_{n}}}{\sum_{n=1}^{18} 10^{0.1 L_{n}}}\right) \text {, }
$$

where $L_{n}$ is normalized A-weighted sound pressure level of traffic noise in the n-th one-third octave frequency band (from $100 \mathrm{~Hz}$ to $5 \mathrm{kHz}$ ); [16].

The standard method applied in the research has been widely described in [15]. The measurements have been carried out for a 4-m high sound barrier constructed of sound reflective panels. On the top edge of the barrier, 10-m long segments have been prepared for installation of added devices of three various shapes (see Fig. 2). These prototypes were delivered to the test.

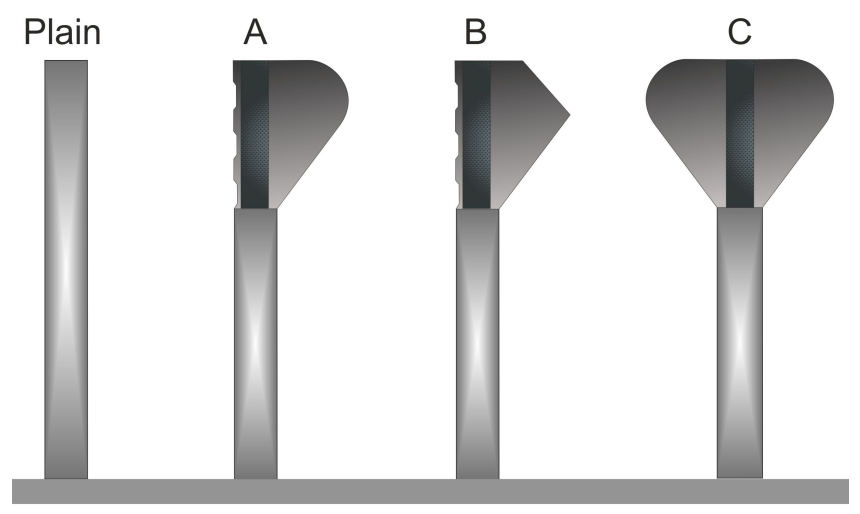

Fig. 2. Shapes of the tested added devices.

Measurement results for three variants of the added devices are presented in Fig. 3. The plots show the sound level differences from the reference measurement (without the barrier) and measurement in given observation points in one-third octave bands for frequencies from $100 \mathrm{~Hz}$ to $5000 \mathrm{~Hz}$. The final result of the described study is the specification of a single-number diffraction index difference, determined separately for each type of the added device. 


\section{Experimental studies}
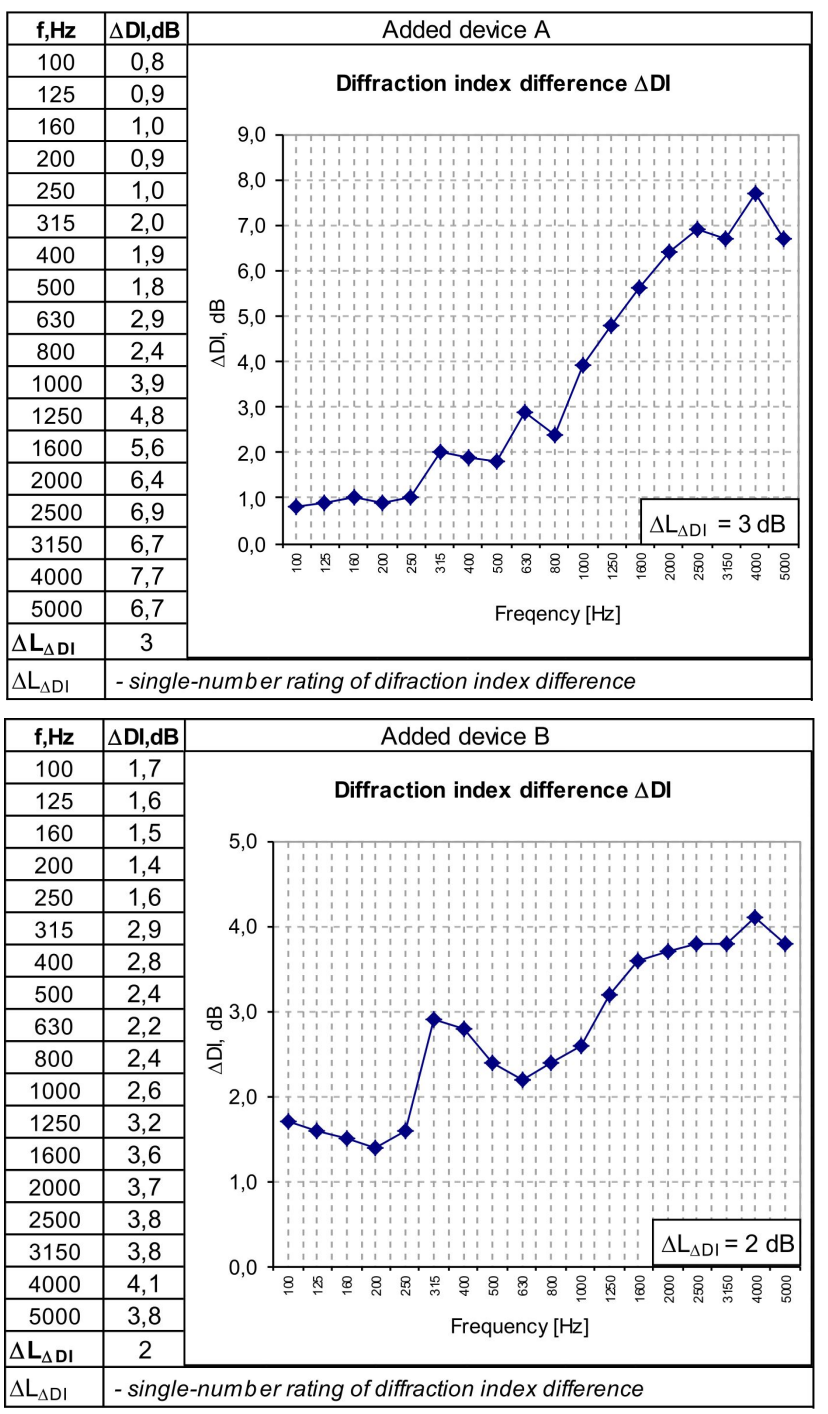

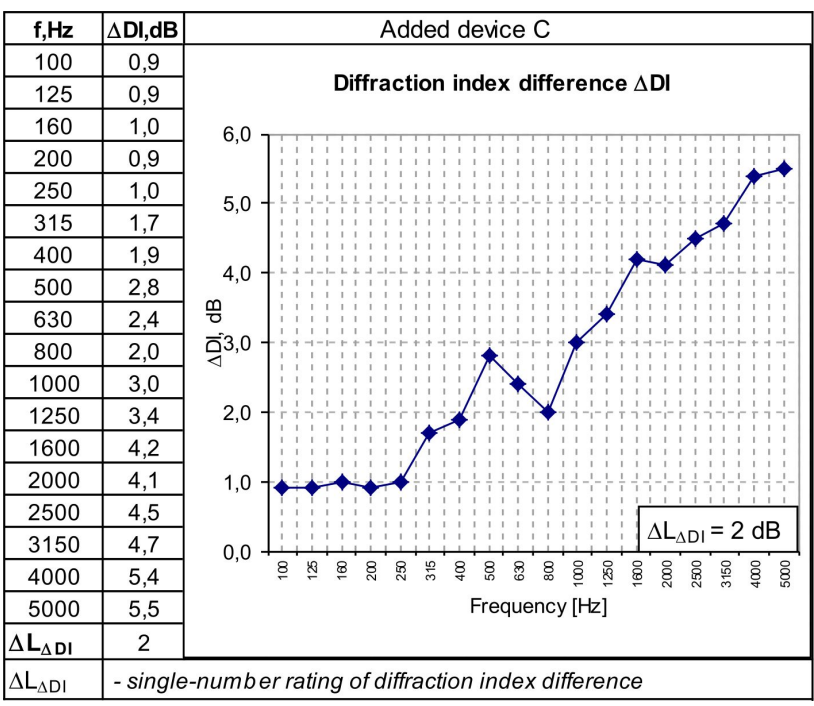

Fig. 3. Diffraction indices difference in one-third octave bands for the tested types of added devices.
The main research problem is getting to know the sound level distribution in the area near the top edge of the barrier both for the plain edge and the edge with added device. For the barrier variants studied earlier with various edge devices, the impulse responses have been determined in the modified arrangement of measurement points. The general layout of the loudspeaker and measurement point locations is shown in Fig. 4. Measurements have been carried out using the same procedure as for the diffraction index difference studies.
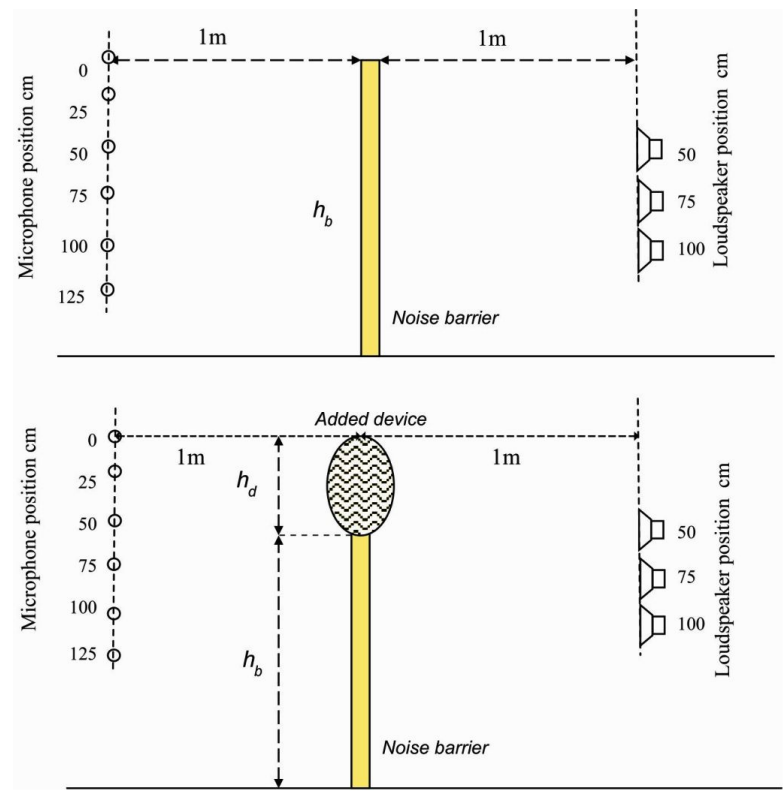

Fig. 4. Layout of the loudspeaker and measurement point locations.

The impulse responses have been registered in 6 measurement points located at 1-m distance from the barrier, in microphone positions located $0 \mathrm{~cm}, 25 \mathrm{~cm}, 50 \mathrm{~cm}, 75$ $\mathrm{cm}, 100 \mathrm{~cm}$, and $125 \mathrm{~cm}$ below the top edge of the barrier. The measurements have been repeated 3 times for three different locations of the sound source $(50 \mathrm{~cm}, 75 \mathrm{~cm}$, and $100 \mathrm{~cm}$ below the top edge of the barrier). Then, the same series of measurements have been carried out on the barrier sections where the added devices of various types (A, B, or C) have been installed. The reference level has been modified (the level $0 \mathrm{~cm}$ has been attributed to the top of the barrier with the added devices - 4.0 $+0.44 \mathrm{~m}=4.44 \mathrm{~m}$ ) both for the microphone and loudspeaker locations. Once more, 3 series of measurements have been carried out - for 6 microphone locations and 3 sound source locations. The registered samples of the impulse responses have been analyzed. The acoustic pressure level differences have been calculated in octave frequency bands between $125 \mathrm{~Hz}$ and $4000 \mathrm{~Hz}$, for the barrier with plain top edge and with added devices installed. Average values of effectiveness have been determined for each added device and for each combination of 


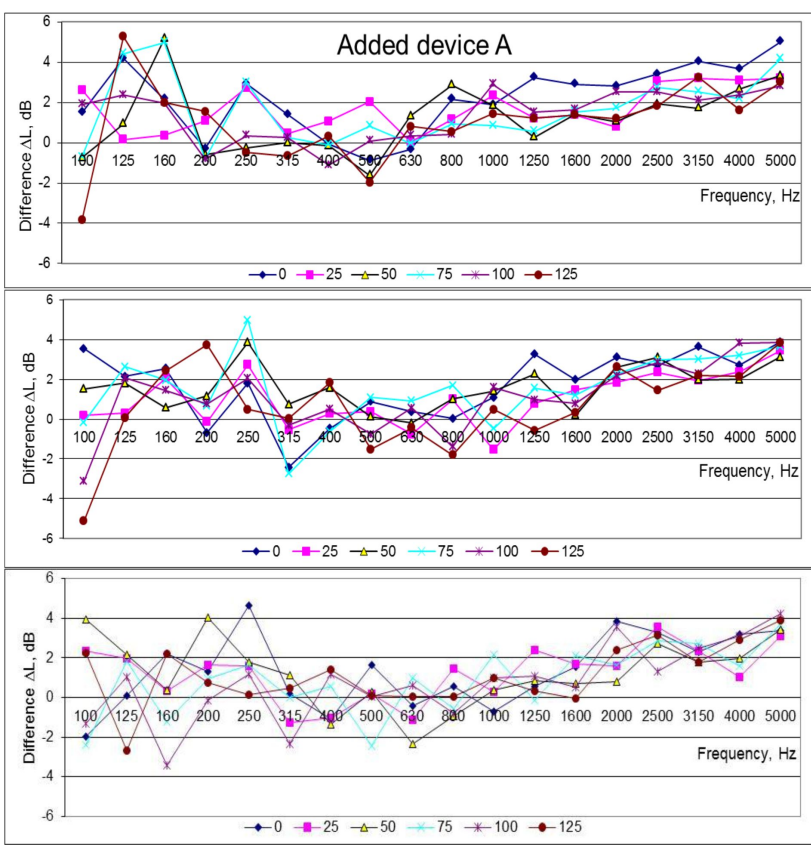

Fig. 5. Added device A. Plots of average effectiveness differences for barrier with plain top edge and with added device installed, various sound source locations $(50 \mathrm{~cm}, 75 \mathrm{~cm}, 100 \mathrm{~cm})$, and various measuring microphone locations with respect to the top edge of the barrier $(0-125 \mathrm{~cm})$.

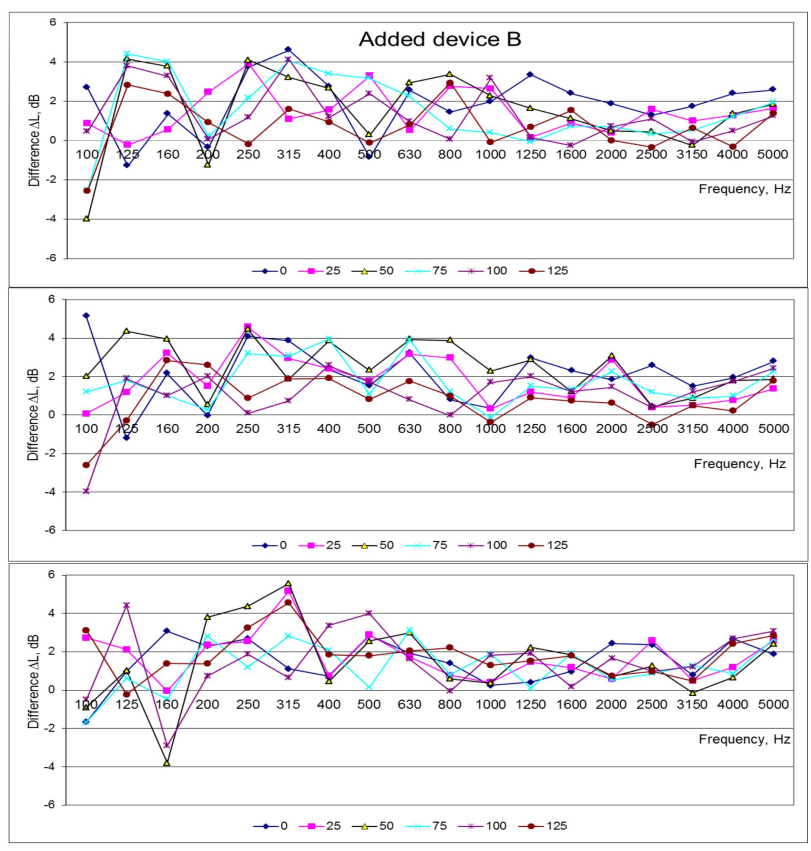

Fig. 6. Added device B. Plots of average effectiveness differences for barrier with plain top edge and with added device installed, various sound source locations $(50 \mathrm{~cm}, 75 \mathrm{~cm}, 100 \mathrm{~cm})$, and various measuring microphone locations with respect to the top edge of the barrier $(0-125 \mathrm{~cm})$.
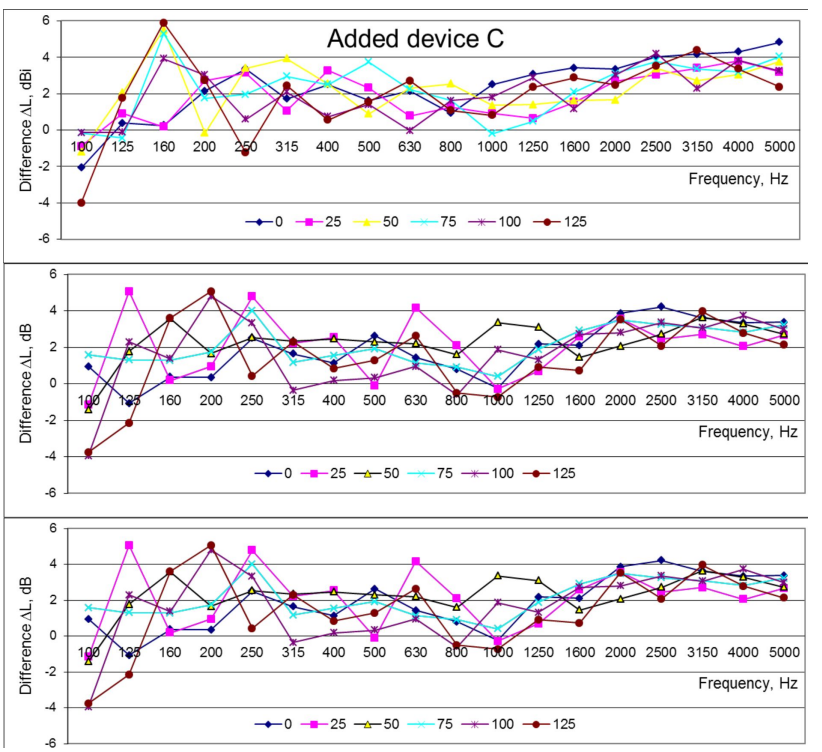

Fig. 7. Added device C. Plots of average effectiveness differences for barrier with plain top edge and with added device installed, various sound source locations $(50 \mathrm{~cm}, 75 \mathrm{~cm}, 100 \mathrm{~cm})$, and various measuring microphone locations with respect to the top edge of the barrier $(0-125 \mathrm{~cm})$.

the loudspeaker-reception point location. The measurement and calculation results for individual added devices of types A, B, and C are presented in Figs. 5-7.

\section{Discussion of the results}

The values of the single-number rating of the diffraction index differences are about $2 \mathrm{~dB}$ for type $\mathrm{B}$ and $\mathrm{C}$ added devices and about $3 \mathrm{~dB}$ for type $\mathrm{A}$ added device. The values are not impressive which means that the additional reduction of the noise level by the barrier with added devices installed is not significant. The earlier studies have shown that the octagon-shaped added device of comparable size exhibited diffraction index difference equal to $0 \mathrm{~dB}$. Much higher indices have been determined for added devices of complex shapes with considerable area of absorbing surfaces (e.g. so called deer antlers).

The differences in sound pressure levels for the acoustic wave transmitted over a plain barrier and the barrier with top edge added devices installed are shown in Figs. 5-7. The plots show that in the area close to the barrier (measuring distance $1 \mathrm{~m}$ ), there is often a reduction of the sound level for barrier with edge added devices in comparison to plain top edge barrier. Smaller reductions of sound levels are encountered for lower frequencies, in the $100 \mathrm{~Hz}$ to $1000 \mathrm{~Hz}$ range, while higher reductions are observed for higher frequencies. The highest reduction of the sound level is exhibited by the A-type added device. For the C-type device and for loudspeaker locations $75 \mathrm{~cm}$ and $100 \mathrm{~cm}$ below the barrier edge, there are increases in the sound level values of about $1 \mathrm{~dB}$ for frequencies around $500 \mathrm{~Hz}$. 
These differences depend on the mutual locations of the sound source and the observation point. In Table below one can find the obtained values of effectiveness for type A, B, and C added devices in comparison to the plain top edge barrier. The values have been weighted according to the normalized road traffic noise spectrum.

\section{TABLE}

Average effectiveness of added devices corrected to the road traffic noise (with application of normalized road traffic noise spectrum) for various locations of the sound source).

\begin{tabular}{|c|c|c|c|c|}
\hline \multirow{3}{*}{\multicolumn{2}{|c|}{$\begin{array}{l}\text { Added } \\
\text { device }\end{array}$}} & \multirow{2}{*}{\multicolumn{3}{|c|}{$\begin{array}{c}\text { Average effectiveness of added devices } \\
\text { weighted according to the normalized } \\
\text { road traffic noise spectrum }[\mathrm{dB}]\end{array}$}} \\
\hline & & & & \\
\hline & & \multicolumn{3}{|c|}{ Sound source below the barrier's top edge } \\
\hline Added & $\mathrm{A}$ & 4.8 & 4.8 & 4.5 \\
\hline device & B & 4.4 & 4.9 & 5.4 \\
\hline type & $\mathrm{C}$ & 4.3 & 3.0 & 2.4 \\
\hline
\end{tabular}

The measurements in the field of direct interaction area of the modified barrier edge indicate that there is a possibility of sound level reduction by a few decibels. The noise barriers are most frequently applied to provide noise protection for buildings that are located in further distance, where the strong effect of the diffraction phenomena is not observed. The studies involving the acoustic field modeling and auralization techniques may contribute much to the information concerning distributions of acoustic field parameters behind acoustic barriers. Visualization of the sound field may be helpful in proper selection of solutions for the barrier construction.

\section{Conclusions}

Intrinsic characteristics of sound diffraction of added devices (Fig. 2) were assessed according to the European technical specification [15]. The single-number rating of the diffraction index difference is $3 \mathrm{~dB}$ for the type $\mathrm{A}$ added device and $2 \mathrm{~dB}$ for type $\mathrm{B}$ and $\mathrm{C}$ added devices. The values of effectiveness for barriers with all types of added devices in comparison to the plain top edge barrier were presented in Table. The difference resulting from the use of added device is of the order of $3-5 \mathrm{~dB}$. This shows the changes that is caused by added devices in the sound field near the top edge of the screen.

The experimental results and the literature review show that the edge added devices have some effect on the acoustic field distribution behind the barrier. The effect depends on the structure geometry (shape) and the material properties of the added device. The investigated structures do not contribute significantly to changes in the noise reduction in the protected area. Barrier structures that have a strong effect on the sound field behind the barrier have to be significantly extended both in horizontal and vertical directions.

\section{Acknowledgments}

The study has been performed within the statutory research of AGH University of Science and Technology, Department of Mechanics and Vibroacoustics (project No. 11.11.130.955).

\section{References}

[1] J. Kompała, J. Martyka, M. Majer, Proceedings 7th Forum Acusticum 2014 Krakow, FA2014, Krakow 2014.

[2] J. Piechowicz, Acta Phys. Pol. A 119, 1040 (2011).

[3] J. Piechowicz, Mech. and Control 29, 179 (2010).

[4] D.J. Oldham, Ch.A. Egan, Appl. Acoust. 72, 803 (2011).

[5] S. Sakamoto, H. Tachibana, Proceedings 18th ICA, Kyoto 2004, p. 525.

[6] S. Weyna, Proceedings 1st Seminar Environmental Protection against Noise 2013 Krakow, Department of Mechanics and Vibroacoustics, Kraków 2013 (in Polish), p. 41.

[7] M. Garai, P. Guidorzi, Proceedings Acoustics 2008, Paris 2008

[8] M. Garai, P. Guidorzi, J Acoust. Soc. Am. 108, 1054 (2000).

[9] G.R. Watts, P.A. Morgan, M. Surgand, J Sound Vib. 274, 669 (2004).

[10] M. Karimi, D. Younesian, J Low Freq. Noise $V A$ 33, 357 (2014).

[11] Z. Engel, J. Piechowicz, L. Stryczniewicz, Fundamentals of industrial vibroacoustics, AGH, Kraków 2003 (in Polish).

[12] A. Ozga, Acta Phys. Pol. A 123, 1034 (2013).

[13] M. Jabłoński, A. Ozga, Distribution of random pulses acting on a vibrating system as a function of its motion, AGH University of Science and Technology Press, Kraków 2013.

[14] J.B. Keller, J Opt. Soc. Am. 62, 116 (1962).

[15] DS./CEN/TS 1793-4; Road traffic noise reducing devices - Test method for determining the acoustic performance - Part 4: Intrinsic characteristics In situ values of sound diffraction.

[16] PN-EN 1793-3:2001 Road traffic noise reducing devices. Test method for determining the acoustic performance. Normalized traffic noise spectrum. 